\title{
Supporting Information for "Microengineered materials with self-healing features for soft robotics"
}

\author{
Vardhman Kumar ${ }^{1}$, Ung Hyun Ko ${ }^{1}$, Yilong Zhou ${ }^{1}$, Jiaul Hoque ${ }^{1}$, Gaurav Arya ${ }^{1}$, and Shyni \\ Varghese $^{1}$
}

${ }^{1}$ Affiliation not available

February 23, 2021

\section{Details of the computational model for balloon actuators in the hindwings:}

\section{Governing equations}

Fluid flow: As the gravitational force is negligible compared to the pressure generated by the water balloon, the time-dependent flow field in the microchannel is governed by the conventional continuity and momentum equations,

$\nabla \cdot \mathbf{u}=0$

$\rho_{f}\left(\frac{\partial \mathbf{u}}{\partial t}+\mathbf{u} \cdot \nabla \mathbf{u}\right)=-\nabla p+\eta \nabla^{2} \mathbf{u}$,

where $\rho_{f}, p, \eta$ and $\mathbf{u}$ are the fluid density, pressure, viscosity and velocity vector, respectively.

Solid mechanics: Since PDMS and the Ecoflex membrane endure large deformations, the second PiolaKirchhoff stress tensor was used in the governing equations as,

$\rho_{m} \frac{\partial^{2} \mathbf{x}}{\partial t^{2}}=\nabla \cdot(\mathrm{FS})^{T}$

$\mathbf{F}=\mathbf{I}+\nabla \mathrm{x}$,

$\mathbf{S}=2 \frac{\partial W_{s}}{\partial \mathbf{C}}$,

where $\mathbf{x}$ is the displacement vector, $\rho_{m}$ is the material density, $\mathbf{F}$ is the deformation gradient, $\mathbf{S}$ is the second Piola-Kirchhoff stress tensor, $\mathbf{I}$ is the identity tensor, $W_{s}$ is the strain-energy function, and $\mathbf{C}=\mathbf{F}^{T} \mathbf{F}$ is the right Cauchy-Green deformation tensor. The Cauchy stress $\sigma$ can be reconstructed in terms of $\mathbf{S}$.

Since PDMS and Ecoflex membranes are hyperelastic materials, suitable strain-energy functions are needed to describe the nonlinear relationship between the stress and strain. For PDMS, the strain-energy function can be expressed by the Mooney-Rivlin model(Mooney, 1940; Rivlin, 1948) as,

$W_{s}=\sum_{i, j=0}^{n} C_{i, j}\left(I_{1}-3\right)^{i}\left(I_{2}-3\right)^{j}+\frac{1}{2} K\left(J_{\mathrm{el}}-1\right)^{2}$

where $I_{1}$ and $I_{2}$ are the first and second invariant of the left isochoric Cauchy-Green deformation tensor, $K$ is the bulk modulus, $J_{\mathrm{el}}$ is the elastic Jacobian, and $C_{i, j}$ are material parameters.

In addition, the strain-energy function for Ecoflex membrane was represented by the Ogden material model(Ogden, 1972) as,

$W_{s}=\sum_{p=1}^{n} \frac{\mu_{p}}{\alpha_{p}}\left(\lambda_{1}^{\alpha_{p}}+\lambda_{2}^{\alpha_{p}}+\lambda_{3}^{\alpha_{p}}\right)+\frac{1}{2} K\left(J_{\mathrm{el}}-1\right)^{2}$

where $\lambda_{i}(i=1,2,3)$ are the principal stretches, and $\mu_{p}$ and $\alpha_{p}$ are material constants. 
Computational domain and boundary conditions: The out-of-plane bending motion of the microchannel takes place in the lateral direction. Therefore, a 3D cuboid domain was chosen for the study set up as shown in Figure S3a, where only a length of $12 \mathrm{~mm}$ in the lateral direction and $3 \mathrm{~mm}$ in the side direction were considered to capture the main dynamic motions of the channel so as to reduce computational cost. This model consists of two layers representing the PDMS and Ecoflex membrane. The fluid domain was embedded into the space between the PDMS and Ecoflex membrane layers, as shown in Figure S3a, where the length $L$ is an adjustable parameter. Note that the balloon expansion mainly appears in the rectangular fluid domain and the inlet tube used in the experimental device only serves as a passage to inject water into the domain; therefore, the part of tube has not been included in the computational domain. Thus, the fluid inlet has been directly assigned to the right end of the rectangular domain. The following boundary conditions were prescribed:

$<$ Fluid flow $>$

Inlet: volume flow rate $V_{0}$;

All other walls: no slip with $\mathbf{u} \cdot \mathbf{n}=0$;

Initial conditions: $\mathbf{u}=0$ and $p=0$.

$<$ Solid mechanics (PDMS and membrane) $>$

Right ends: fixed constraint with $\mathbf{x}=0$;

All outer boundaries: free boundaries with zero stress.

Initial conditions: $\mathbf{x}=0$ and $\frac{\partial \mathbf{x}}{\partial t}=0$.

Multiphysics: This fluid-structure interaction was assigned to the interfaces between the fluid domain and solid mechanics.

Numerical approach: The dynamic bending motion of the cuboid microchannel in Figure S3a was simulated in COMSOL 5.3a. Equation 1 and 2 were solved in the Laminar Flow module, and the Moving Mesh was applied for the fluid domain. The Solid Mechanics module was used to solve for the structure deformation from Equation 3-7. Within the module, the five parameters Mooney-Rivlin material model (Equation 6) was used to calculate the strain-energy function for PDMS while Ogden material model (Equation 7) was used for Ecoflex membrane. The Mooney-Rivlin parameters for PDMS was used from a previous work(Kim et al., 2011). The Ogden material constants for Ecoflex00-30 membrane determined from the mechanical measurements were used in the simulation. The Fluid-Structure Interaction was assigned to the coupled interfaces between fluid flow and solid mechanics. A time-dependent study was added to solve the model. All the values of the physical and geometrical parameters are summarized in Table 1.

\section{Supplementary Figures}




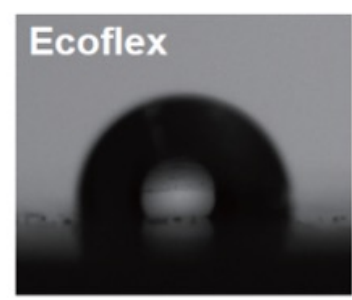

$96.1^{\circ} \pm 3.07^{\circ}$

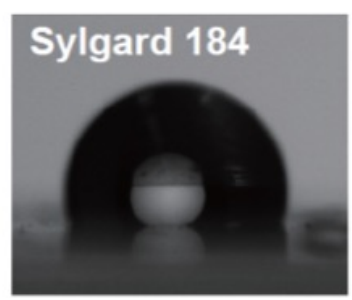

$106.3^{\circ} \pm 1.12^{\circ}$

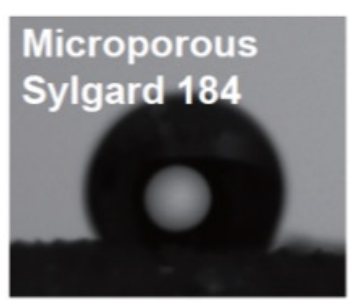

$126.2^{\circ} \pm 5.47^{\circ}$

Figure S1. The hydrophobicity of materials was quantified using contact angle measurement. The contact angles of Ecoflex, Sylgard 184, and microporous Sylgard 184 were $96.1^{\circ} \pm 3.07^{\circ}, 106.3^{\circ} \pm 1.12^{\circ}, 126.2^{\circ} \pm$ $5.47^{\circ}$ respectively.

a

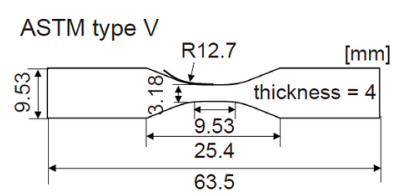

C

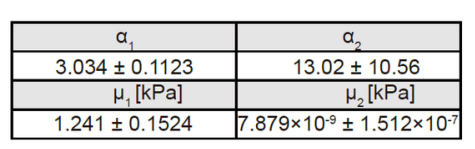

b $\quad[\mathrm{kPa}]$

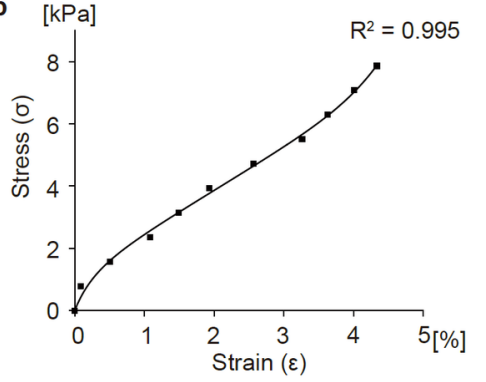

Figure S2. Mechanical test/analysis of Ecoflex 00-30.(a ) Tensile test sample dimension according to the ASTM type V standard. (b ) Curve fitting graph of Ecoflex 00-30 stress-strain data with Ogden model. (c ) Obtained Ogden model parameters from curve fitting data.
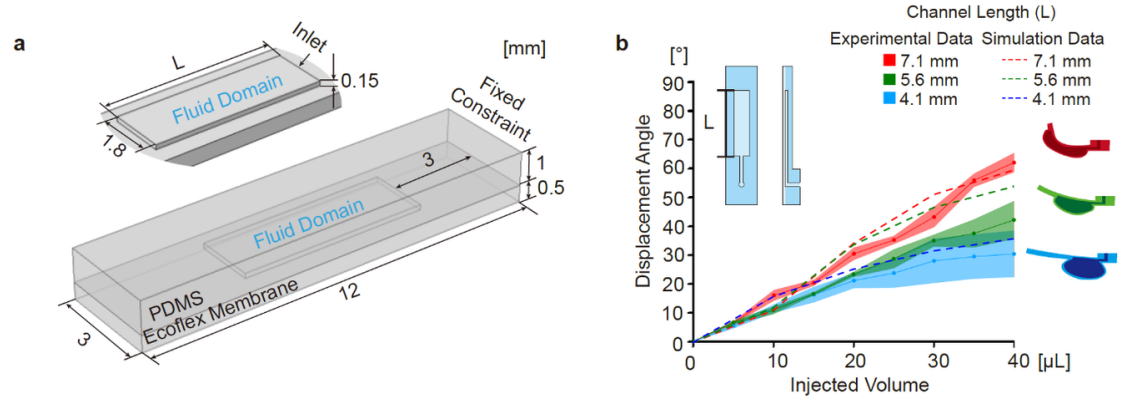

Figure S3. Simulation of balloon actuator for optimizing channel dimensions. (a ) Isometric view 
of the 3D cuboid domain of balloon actuator. The fluid domain is embedded into the space between the PDMS and membrane layers, as highlighted in the inset. (b ) Comparison of experimental (solid line) and simulation (dash line) results of displacement angle $(\vartheta)$ with different rectangular channel lengths: 4.1, 5.6, and $7.1 \mathrm{~mm}$.
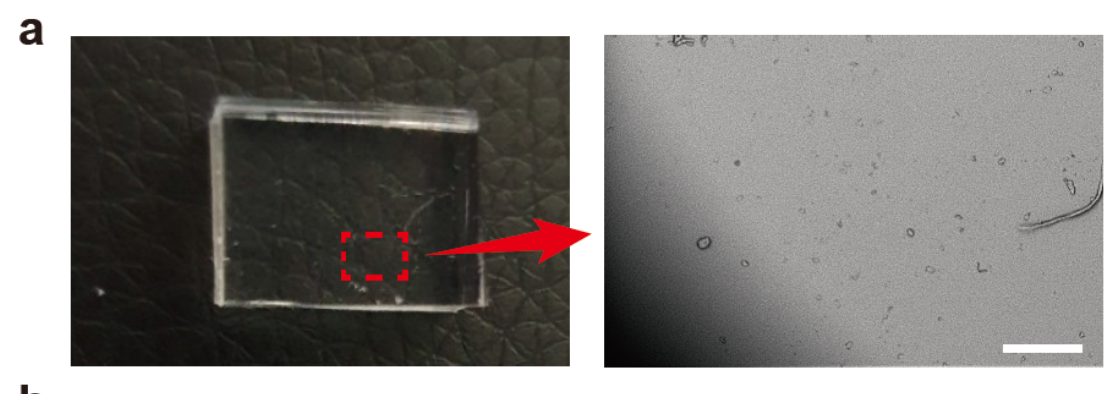

b

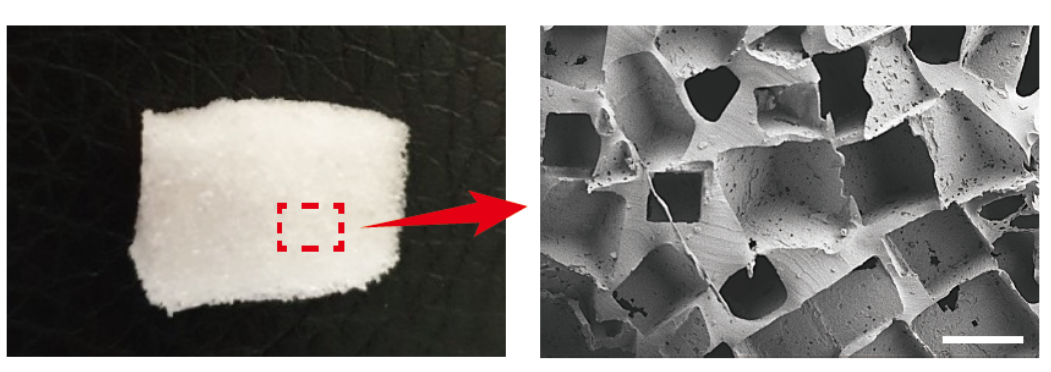

Figure S4. Scanning Electron Microscope images of PDMS microstructure. (a ) Bare PDMS slab has a clear surface without any microstructure. (b ) In contrast, microporous PDMS substrate shows interconnected salt crystal-shaped pores. Scale bar: $250 \mu \mathrm{m}$. 


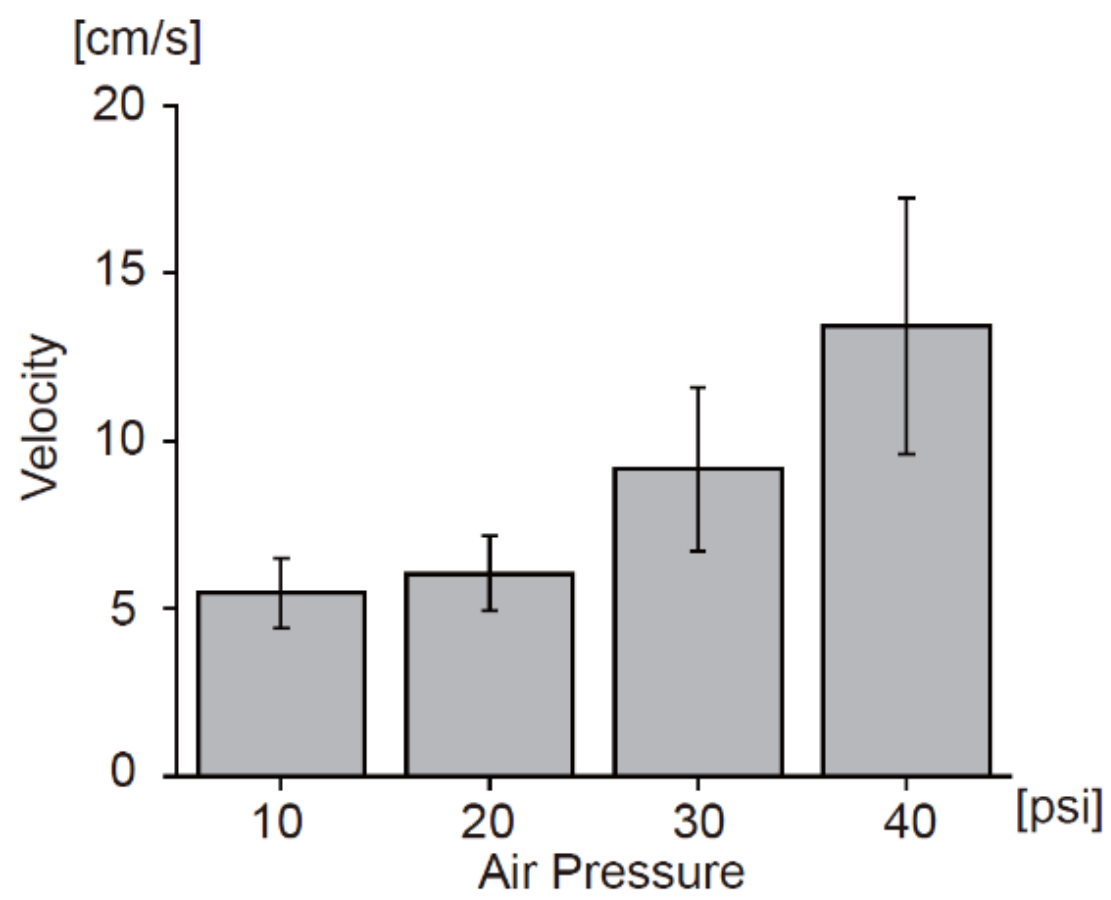

Figure S5. DraBot velocity in various pneumatic input pressure.Velocity increases from $\sim 5.5 \mathrm{~cm} / \mathrm{s}$ to $13.4 \mathrm{~cm} / \mathrm{s}$ when air pressure is increased from 10 to 40 psi.
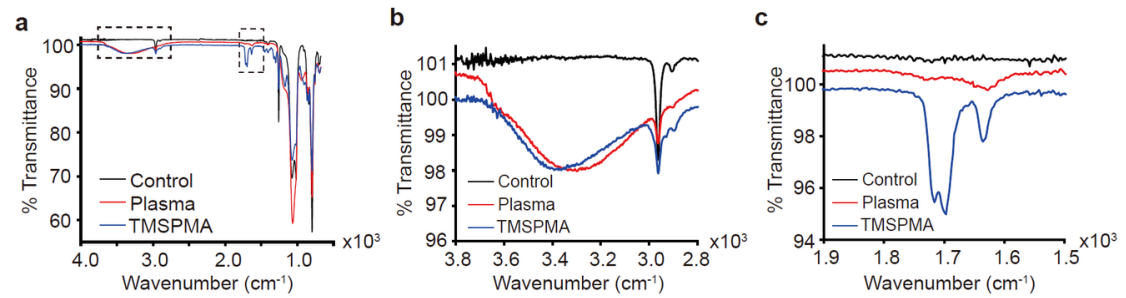

Figure S6. FTIR spectra of bare, plasma treated, TMSPMA modified PDMS surface. (a )The total FTIR graph in $4000^{\sim} 500 \mathrm{~cm}^{-1}$ wavenumber. (b ) \% transmittance change at $3200^{\sim} 3400 \mathrm{~cm}^{-1}$ following surface oxygen plasma treatment . Peak at $\sim 1700 \mathrm{~cm}^{-1}$ corresponding to a $\mathrm{C}=\mathrm{C}$ bond is observed in TMSPMA-modified PDMS surface. (c ) Control: bare PDMS surface. Plasma: PDMS modified by oxygen plasma. TMSPMA: plasma-treated PDMS reacted with TMSPMA.

\section{Table}

Table S1. Physical and geometric parameters used in the numerical simulation 


\section{Interactive Figure}

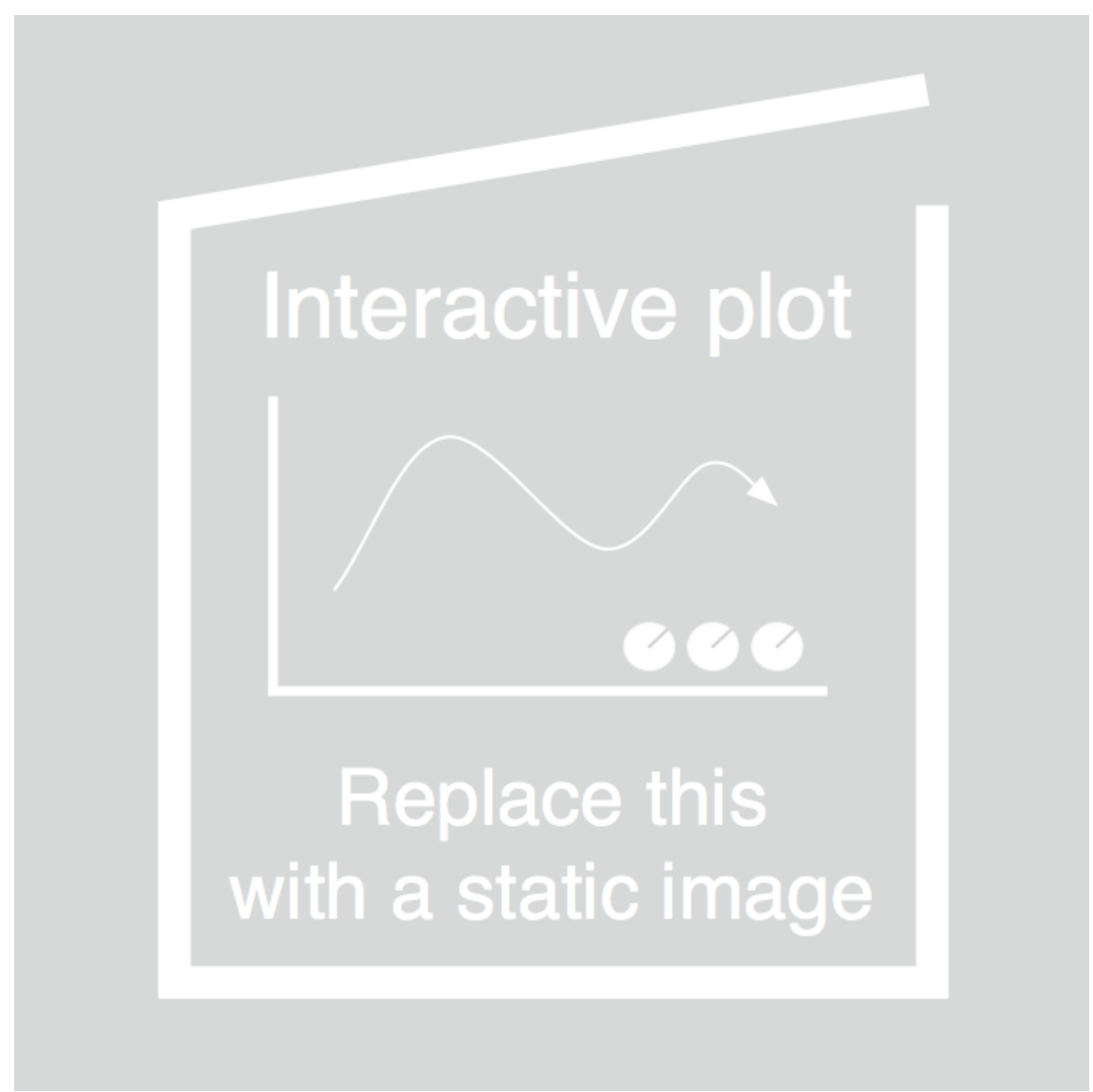

Interactive Figure S1. 3D CAD model of DraBot. Red part represents Sylgard 184, blue part represents the Ecoflex 00-30 and the white structures are the microporous Sylgard 184.

\section{Videos}

Rich media available at https://youtu.be/TKwdns254c4

Movie S1. Ecoflex 00-30 membrane inflates significantly, causing the entire PDMS structure to bend. The size of the rectangular channel in the balloon actuator is $7.1 \mathrm{X} 1.8 \mathrm{X} 0.15 \mathrm{~mm}$ (length X width X height). 
Rich media available at https://youtu.be/-UdIw_zTxjc

Movie S2. The maximum bending angle and the increase in rate of angle of the balloon actuator is proportional to channel length.

Rich media available at https://youtu.be/eaXm7_PYVG4

Movie S3. Flapping/flexing motion of each hindwing is controlled separately by two syringes, and the independent flexing of each hindwing allows the DraBot to change traveling direction.

Rich media available at https://youtu.be/UhOPHEpNMSg

Movie S4. Both hindwings flexed upward to unblock the air outlets of both forewings, leading to unidirectional airflow and forward propulsion. Maintaining the upward flap of the hindwings results in a straight motion.

Rich media available at https://youtu.be/j_Bxp68Dfpk

Movie S5. Left hindwing flexing generates net torque, resulting the bot to turn rightwards. A sustained right-turn signal leads to continuous clockwise motion.

Rich media available at https://youtu.be/tje5JFXd80c

Movie S6. Net torque, generated by the right hindwing flexing, makes the bot turn leftwards. A sustained left turn signal leads to continuous counterclockwise motion.

Rich media available at https://youtu.be/dOwAy8go2FI

Movie S7. Complex motion achieved by using multiple input signal in series.

Rich media available at https://youtu.be/pL81NHX6Qv0

Movie S8. pH-sensitive self-healing of the A6ACA hydrogel facilitates welding of fore- and hindwing upon exposure to acid, which prevents the flapping of hindwing.

Rich media available at https://youtu.be/b8QjHZxDJok 
Movie S9. Welding of fore and hindwings (left side) block the air outlet on left forewing, which results in DraBot making a continuous left turn, despite receiving an input signal for forward motion.

Rich media available at https://youtu.be/OXG_JDVqsEQ

Movie S10. When exposed to a temperature above $37^{\circ} \mathrm{C}$, the wing color changes from red-to-yellow, and reverts back when exposed to room temperature.

Rich media available at https://youtu.be/j1pQ3upYYvg

Movie S11. The Sylgard 184 hydrophobicity along with the microstructure mediated increase in surface area allows microporous structure to imbibe hydrophobic oil while no such absorption is observed with water.

Rich media available at https://youtu.be/hBDd5QTUAno

Movie S12. The microporous PDMS structures present at the wings and abdomen absorb oil from the water when encountered. For better visualization, colorless Sylgard 184 was used to fabricate DraBot. The red color shows the absorption of oil.

\section{References}

A Theory of Large Elastic Deformation. (1940). Journal of Applied Physics, 11(9), 582-592. https: //doi.org/10.1063/1.1712836

Large elastic deformations of isotropic materials IV. further developments of the general theory. (1948). Philosophical Transactions of the Royal Society of London. Series A Mathematical and Physical Sciences, 241(835), 379-397. https://doi.org/10.1098/rsta.1948.0024

Large deformation isotropic elasticity - on the correlation of theory and experiment for incompressible rubberlike solids. (1972). Proceedings of the Royal Society of London. A. Mathematical and Physical Sciences, 326(1567), 565-584. https://doi.org/10.1098/rspa.1972.0026

Measurement of nonlinear mechanical properties of PDMS elastomer. (2011). Microelectronic Engineering, 88(8), 1982-1985. https://doi.org/10.1016/j.mee.2010.12.108 